\title{
Determinación de la capacidad auditiva a efectos de los requerimientos exigidos para la conducción de vehículos
}

\section{Determination of the hearing for the purposes of required requirements for driving}

\section{Ana Gómez Álvarez', José Luis de la Fuente Madero', Antonio Domínguez Muñoz', Javier de Pro Bueno', Elvira Cabuchola Fajardo', Santiago Cabuchola Moreno'}

1. Unidad Médica del Equipo de Valoración de Incapacidades. Instituto Nacional de la Seguridad Social. Dirección Provincial de Málaga. España

2. Servicio de Otorrinolaringología. Hospital Puerta del Mar. Cádiz. España

Recibido: 11-02-15

Aceptado: 27-02-15

\section{Correspondencia}

José Manuel Vicente Pardo

Pescadores Gran Sol s/n

San Sebastián 2001 1, Gipuzkoa. España.

Correo electrónico: jose-manuel.vicente@seg-social.es

Resumen

En España existe una regulación reglamentaria que vela por que el conductor se encuentre en las adecuadas condiciones psicofísicas intentando controlar y reducir los accidentes de tráfico.

La normativa española actual exige, para la obtención del permiso o licencia de conducción, entre otros requisitos, someterse a un reconocimiento médico que evidencie que el interesado en conseguirlo no padece enfermedades o mermas en sus cualidades que aumenten el riesgo en la conducción de vehículos. El papel del médico se hace insustituible ya que como resultado del reconocimiento y tras conocer las circunstancias individuales de cada conductor se podrá determinar su capacidad para la conducción y por tanto permitirá o no la obtención o prórroga del permiso de conducción.

La regulación legal en esta materia pretende disminuir la accidentabilidad en las carreteras como método de prevención primaria y establecer unos criterios uniformes en todo el territorio nacional fomentando la armonización.

Conclusiones: Sería conveniente que en el propio Reglamento General de Conductores se estableciera el procedimiento concreto para calcular las limitaciones en aquellos casos en que existan varios métodos de cálculo posibles, como es el caso de la agudeza auditiva. Es preciso asumir los criterios y conclusiones del resultado de la valoración de la aptitud para conducir según los requisitos exigidos en el RGC para en general aminorar las controversias que se susciten caso de discrepancia, y en estos supuestos arbitrar procedimientos de actuación. Si aceptamos que para la valoración de la capacidad laboral en determinadas profesiones (aquellas que incluyen entre sus requerimientos fundamentales la conducción de vehículos de motor), uno de los factores a considerar es lo dispuesto en el Anexo IV del RGC, sería importante que hubiera consenso en la interpretación del mismo buscando la homogeneización y armonización de criterios entre los distintos agentes con competencias en la valoración de incapacidades, de cara a seguir unos criterios homogéneos en todo el territorio nacional y disminuir la variabilidad interobservador.

Med Segur Trab (Internet) 2015; 61 (238) 78-85

Palabras clave: Déficit auditivo, conducción de vebículos, valoración capacidad laboral. 


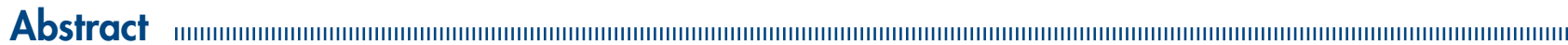

Conclusions: It would be desirable that the General Drivers Regulations own concrete procedure be established to calculate the limitations in cases where there are several possible methods of calculation, such as auditory acuity. It's necessary to take the criteria and conclusions on the outcome of the assessment of fitness to drive according to the requirements of the RGC to generally reduce disputes arising case of discrepancy, and in these cases arbitrate operating procedures. If we accept that for the assessment of work capacity in certain professions ( those that include among its key requirements driving motor vehicles), one factor to consider is the provisions of Annex IV of the RGC, it would be important that there consensus on the interpretation of it looking homogenization and harmonization of approaches among actors with competence in the assessment of disabilities, in order to follow homogeneous criteria throughout the national territory and decrease interobserver variability.

Med Segur Trab (Internet) 2015; 61 (238) 78-85

Keywords: Hearing impaired, driving, capacity assessment, disability, work capacity assessment. 


\section{INTRODUCCIÓN}

En España existe una regulación reglamentaria que vela por que el conductor se encuentre en las adecuadas condiciones psicofísicas intentando controlar y reducir los accidentes de tráfico.

La normativa española actual exige, para la obtención del permiso o licencia de conducción, entre otros requisitos, someterse a un reconocimiento médico que evidencie que el interesado en conseguirlo no padece enfermedades o mermas en sus cualidades que aumenten el riesgo en la conducción de vehículos. El papel del médico se hace insustituible ya que como resultado del reconocimiento y tras conocer las circunstancias individuales de cada conductor se podrá determinar su capacidad para la conducción y por tanto permitirá o no la obtención o prórroga del permiso de conducción. Huelga decir que si tras dicho reconocimiento médico se derivara la conclusión de no aptitud, no sería posible expedir la mencionada licencia.

Por tanto, la regulación legal en esta materia, además de pretender disminuir la accidentabilidad en las carreteras como método de prevención primaria cuyo objeto es aumentar la seguridad vial, tiene como efecto no menos importante establecer unos criterios uniformes en todo el territorio nacional, de manera que todos los individuos que deseen solicitar el permiso de conducción o prorrogarlo, tengan que cumplir unos criterios uniformes de aptitud, disminuyendo la variabilidad y fomentando la armonización de criterios en el territorio en el que dicha norma es aplicable. De esta forma, se impide que las personas aquejadas por determinadas limitaciones puedan obtener o prorrogar la licencia en ningún lugar del territorio nacional.

En España, el Real Decreto 818/2009, de 8 de mayo, por el que se aprueba el Reglamento General de Conductores (RGC), y más concretamente su anexo IV, modificado por la Orden PRE/2356/2010 de 3 de septiembre, es la normativa de referencia por la que se establecen las aptitudes psicofísicas requeridas para obtener o prorrogar la vigencia del permiso o de la licencia de conducción, es decir, señala las enfermedades y deficiencias que serán causa de denegación, adaptaciones, restricciones de circulación y otras limitaciones en la obtención o prórroga del permiso o la licencia de conducción.

Dichas pruebas y exploraciones serán practicadas por un médico en los centros de reconocimiento de conductores (CRC) autorizados, los cuales emitirán un informe de aptitud psicofísica de acuerdo con lo establecido en el Real Decreto 170/2010, de 19 de febrero, por el que se aprueba el Reglamento de centros de reconocimiento destinados a verificar las aptitudes psicofísicas de los conductores.

La aplicación del anexo IV del RGC tiene importantes repercusiones para la vida cotidiana al impedir la conducción de vehículos (conductores del grupo 1), y por supuesto para la vida laboral, por poderse limitar el acceso a licencias de conducción imprescindibles para la realización de muchos trabajos. Tal es el caso de los conductores profesionales (conductores del grupo 2) y conductores del grupo 1, para los que, sin ser considerado profesionales de la conducción, una de las tareas básicas de su profesión es la conducción de vehículos (por ejemplo, agentes comerciales).

Como se ha comentado, el anexo IV del RGC es de obligado cumplimiento en los centros médicos implicados en la expedición de certificados de aptitud para la obtención o prórroga de licencias, pero también, con frecuencia, suele ser tenido en cuenta y ser objeto de consulta en la valoración de la capacidad laboral por aquellos agentes implicados en la misma (aun sin ser vinculante en este otro ámbito) como son los Médicos Inspectores de las Unidades Médicas de los Equipos de Valoración de Incapacidades (UMEVI), Médicos Inspectores de los Servicios Públicos de salud, Médicos del Trabajo... Al ser los médicos de atención primaria la puerta de acceso a la prestación de incapacidad temporal por contingencias comunes y jugar un papel esencial en la determinación de la procedencia de la continuidad o no de la situación de incapacidad temporal, también podría ser este Anexo IV objeto de consulta por ellos. 
Uno de los puntos contemplados expresamente en el RGC es precisamente la función auditiva que permite la percepción de señales acústicas o ruidos siendo precisas unas exigencias mínimas para lograr una conducción segura. Preocupados por la posibilidad de que la descripción contemplada en el RGC para determinar los requerimientos auditivos exigibles pueda ser susceptible de interpretación, deseamos hacer algunas especificaciones para contribuir a la disminución de la variabilidad interevaluador y fomentar el consenso de criterios entre los diversos agentes implicados en la valoración médica de incapacidades en todo el territorio nacional.

\section{OBJETIVOS}

- Mencionar la normativa existente en la que se especifican los requisitos psicofísicos para obtener o prorrogar el permiso o licencia de conducción, de uso obligado en unos casos (médicos de los centros de reconocimiento de conductores) y aconsejable en otros, como los médicos de diferentes ámbitos implicados en la gestión de las prestaciones de la Seguridad Social relacionadas con la incapacidad laboral, esto es, la Incapacidad Temporal (IT) y la Incapacidad Permanente (IP).

- Revisión del apartado 2.1 del Anexo IV del Reglamento General de Conductores, que se refiere a la agudeza auditiva, identificación de posibles causas de confusión y propuestas de mejora.

- Concretar determinados aspectos en la valoración de la capacidad laboral en aquellos profesionales en las que la conducción de vehículos es primordial para el desarrollo de su actividad laboral.

- Proponer el procedimiento para el cálculo del índice de pérdida auditiva combinada para aquellos profesionales de la valoración que usen como instrumento de consulta el Anexo IV del RGC para valorar la capacidad laboral.

\section{NORMATIVA LEGAL VIGENTE MÁS RELEVANTE EN ESTA MATERIA}

- El texto articulado de la Ley sobre Tráfico, Circulación de Vehículos a Motor y Seguridad Vial, aprobado por Real Decreto legislativo 339/1990, de 2 de marzo (y modificada por la Ley 18/2009 de 23 de noviembre), es desarrollado por el Reglamento General de Conductores (RGC), aprobado por el Real Decreto 818/2009 de 8 de mayo, que continúa vigente en la actualidad. En relación al tema que nos ocupa es especialmente relevante el Anexo IV de este RD que fue modificado por la Orden PRE/2356/2010, de 3 de septiembre, por la que se establecen las aptitudes psicofísicas requeridas para obtener o prorrogar la vigencia del permiso o de la licencia de conducción.

- Real Decreto (RD) 170/2010, de 19 de febrero, por el que se aprueba el Reglamento de centros de reconocimiento destinados a verificar las aptitudes psicofísicas de los conductores.

Sin ánimo de ser exhaustivos, destaco los puntos de mayor interés de este último:

En los centros de reconocimientos de conductores (CRC) destinados a verificar las aptitudes psicofísicas de los conductores se realizan las pruebas y exploraciones necesarias para verificar que los interesados en obtener o prorrogar la vigencia del permiso o de la licencia de conducción reúnen las aptitudes psicofísicas necesarias y que no están afectados por alguna de las enfermedades o deficiencias que pueden suponer incapacidad para conducir o la necesidad de establecer adaptaciones, restricciones u otras limitaciones, de acuerdo con la normativa vigente, y más concretamente con el anexo IV del RGC.

En relación con los informes de aptitud psicofísica, el RD 170/2010 determina, entre otros aspectos, la forma en que deberán emitirse y el contenido de cada uno de ellos, de manera que el resultado de la exploración se consignará en un informe que se extenderá 
en el modelo oficial de impreso que se recoge en el anexo II del mencionado RD (bajo el título de "Informe de aptitud psicofísica") y los datos de la exploración efectuada se harán constar en el modelo de historia clínica que se recoge en el anexo III del mismo Real Decreto.

\section{REQUERIMIENTOS AUDITIVOS RECOGIDOS EN ELANEXO IV DEL REGLAMENTO GENERAL DE CONDUCTORES}

El apartado 2 del anexo IV del RGC, bajo la denominación de "Capacidad auditiva", hace referencia a los requerimientos auditivos necesarios para obtener o prorrogar la vigencia del permiso o licencia de conducción. Cabe resaltar que la única exigencia se refiere al umbral auditivo, prescindiendo de otros parámetros que pudieran afectar la audición (diploacusia, distorsiones auditivas...).

En relación con el umbral auditivo, se menciona para el grupo 1 y el grupo 2 que las hipoacusias con o sin audífono, con una pérdida combinada entre los dos oídos de más de $45 \%$ o 35\% respectivamente, obteniendo el índice de esta pérdida mediante una audiometría tonal, impiden la obtención o prórroga de la licencia.

En el caso de que en el grupo 1 no se lleguen a estos niveles auditivos se ofrece como posibilidad para suplir el déficit auditivo añadir espejos retrovisores exteriores a ambos lados del vehículo y espejo interior panorámico. Adaptado de esa forma el vehículo, en el Anexo IV del RGC no se establece suelo auditivo, de lo que se puede deducir que con la mencionada adaptación, al sujeto se le consideraría apto en cualquier nivel de audición (incluso la cofosis).

Pero el cálculo del índice de pérdida combinada (IPC) es fundamental para los conductores del grupo 2, ya que, según el RGC éstos no tienen posibilidad de adaptación.

Sin embargo, el apartado $2 \mathrm{del}$ anexo IV del reglamento no indica el procedimiento para el cálculo de la pérdida auditiva combinada y es éste uno de los aspectos fundamentales que queremos destacar: queremos proponer el uso de aquel procedimiento que nos parece más apropiado para quienes recurren al Anexo IV del RGC como uno de los elementos para valorar la capacidad laboral de los pacientes en los que la conducción de vehículos es tarea básica de su profesión. El cálculo del IPC lo establece el RD 170/2010, de la siguiente forma: se calcula la pérdida tonal media (PTM) de cada oído calculando la media aritmética de los umbrales de audición de las frecuencias de 500, 1000, 2000 y 4000 (es decir, dividiendo entre 4 el resultado de la suma de esos umbrales). De esta forma se determina el oído de mejor audición (oído mejor: OM) y el de peor audición (oído peor: OP). Finalmente el IPC se calcula aplicando la siguiente fórmula: [(PTM OM x7) + PTM OP] / 8

En nuestra opinión, para evitar confusiones sería aconsejable que se indicara el procedimiento de cálculo de la agudeza auditiva en el propio RGC, ya que tal y como aparece en el texto normativo, el lector desconocedor del RD 170/2010, podría sentirse erróneamente impelido a utilizar, por ejemplo, el procedimiento propuesto por el Real Decreto 1971/1999, de 23 de diciembre, de procedimiento para el reconocimiento, declaración y calificación del grado de discapacidad, en el cual se utiliza exactamente la misma expresión (pérdida auditiva combinada), para determinar la discapacidad derivada de esta función, y para cuyo cálculo se defiende un procedimiento distinto. Huelga decir que tras la aplicación de uno u otro el resultado es distinto.

Existen diversos procedimientos propuestos para calcular la pérdida de función auditiva de un modo que tenga en cuenta simultáneamente la capacidad de ambos oídos. Nos encontramos aquí con dos normas diferentes, reguladoras de aspectos diversos de nuestra realidad social, que proponen dos métodos distintos para lograr ese objetivo. Puesto que el resultado obtenido por una u otra vía es diferente, consideramos, en aras de la disminución de la variabilidad interevaluador, necesario consensuar sin margen de 
dudas cuál de ambos métodos es el procedente en nuestro ámbito, circunstancia que se hace necesaria por cuanto el propio RGC no concreta el procedimiento a utilizar.

\section{APLICACIÓN DE LA CITADA NORMATIVA EN LA VALORACIÓN DE LA CAPACIDAD LABORAL}

Después de lo antes expuesto, vamos a centrarnos en la utilidad que para los médicos implicados en la gestión de las prestaciones en materia de incapacidad laboral puede tener el conocimiento de la normativa descrita. Como ya hemos dicho, es de interés tenerla en cuenta para ese colectivo por la contradicción que supondría que estos médicos entendieran la existencia de capacidad laboral en un paciente en el que no se reunieran los requisitos legales expresados en la norma, hecho que, en condiciones normales, conllevaría imposibilidad para prorrogarle el permiso de circulación precisamente por tales causas médicas.

Las profesiones en las que nos vamos a centrar obviamente son aquellas en las que la conducción de vehículos es una tarea fundamental para su desarrollo. En primer lugar los conductores profesionales, es decir, el señalado en el artículo 45 del RGC como grupo II; en segundo lugar, aquellas profesiones en las que, aun no siendo preciso estar en posesión de una licencia de conducción del grupo II (conductores profesionales), sí es absolutamente necesario el manejo de un vehículo (con la consiguiente exigencia de poseer una licencia de conducción del grupo I) como parte fundamental para poder llevar a cabo su trabajo, siendo éste de hecho una de las exigencias por parte del empleador para que el candidato pueda acceder al puesto o mantener su puesto de trabajo. Como ejemplo clásico se encuentran los agentes y representantes comerciales.

A continuación citamos textualmente un párrafo que se refiere a este colectivo, incluido en la última edición de la Guía de Valoración Profesional del INSS: "También existen algunas profesiones en las que un determinado permiso no es obligatorio, aunque en la práctica habitual, en el mercado laboral español, las posibilidades de acceder a dicha profesión están muy limitadas si no se tiene ese permiso administrativo. No se trata de que eventualmente sean solicitados estos permisos, sino de profesiones muy limitadas, en las que casi siempre, el desempeño vaya asociado a dicho permiso. Por ejemplo, hay profesiones como representantes comerciales, que difícilmente se pueden ejercer si no se dispone de carnet de conducir. Así se ha reflejado en las fichas correspondientes, especificando cuál es el permiso que puede ser exigido en esas ocasiones».

Como señala este párrafo, a estos profesionales se les exige disponer de una licencia de conducción para poder desempeñar su profesión. Esto no es previsible que suponga un obstáculo insalvable para la prórroga de la licencia de conducción ya que, como se ha dicho antes, en el grupo I (conducción no profesional) la pérdida auditiva, aunque supere lo indicado en el Anexo IV del RGC puede ser suplido con las adaptaciones que la propia norma propone (espejos exteriores e interior panorámico). Puesto que para estos trabajadores la adaptación del vehículo permite obviar la limitación física, no es previsible que una disminución auditiva aislada, por grave que ésta sea, llegue a condicionar una incapacidad por sí sola. En estos casos sí habría que tener en cuenta (a diferencia del Anexo IV del RGC) otros aspectos de la función auditiva, como son la presencia de otros síntomas, que puedan interferir en la conducción.

En cuanto a los «conductores profesionales», en nuestra valoración, tendremos que tener en cuenta, como en otras patologías, si la situación está o no estabilizada, la imposibilidad de realizar otros tratamientos para mejorar la funcionalidad, la posibilidad de adaptación protésica, la existencia de otros síntomas acompañantes y su repercusión funcional. Vamos a profundizar en algunos de estos puntos:

- Al igual que ocurre en los conductores no profesionales, la existencia de otros síntomas (diploacusia, algiacusia,...) puede limitar la capacidad de conducción, aun cuando este aspecto no esté expresamente contemplado en el RGC. 
- Puesto que el Reglamento de los CRC, aprobado por RD 170/2010, establece como preceptivo un método para el cálculo de la perdida auditiva combinada, entendemos que para valorar la capacidad laboral debe ser el método propuesto por esta norma el que se utilice para concretar las limitaciones a las que se refiere el anexo IV del RGC.

- Dado que el RGC contempla expresamente la posibilidad de que la medición de la agudeza auditiva se haga con audífono, entendemos que si el umbral auditivo mejora con el uso de audioprótesis, la audiometría ha de hacerse con ésta y los cálculos para obtener el IPC deben basarse en los valores así obtenidos.

\section{CONCLUSIONES}

- Como resumen del procedimiento para el reconocimiento de aptitud del permiso de conducción, diremos que el RGC establece que para que la Jefatura Provincial de Tráfico expida el permiso o licencia de conducción solicitada por la persona interesada, es necesario, entre otros requisitos, que ésta reúna las condiciones psicofísicas reflejadas en el anexo IV del mismo RGC, mediante la realización de pruebas de aptitud psicofísica y reconocimientos médicos que dejen constancia de que no existe enfermedad o deficiencia que pueda suponer incapacidad para conducir. Estos reconocimientos médicos se llevan a cabo por médicos de los Centros de Reconocimientos de Conductores, cuyo proceder se encuentra descrito en el Reglamento de los centros de reconocimiento aprobado por el RD 170/2010.

- El Anexo IV del RGC señala las aptitudes psicofísicas necesarias para obtener o prorrogar la vigencia del permiso o licencia de conducción, pero en nuestra opinión, en algunos puntos del mismo no se concretan ni se especifican adecuadamente, lo que puede llevar a distintas interpretaciones de aplicación. Es el caso del punto 2 del mencionado Anexo (capacidad auditiva).

- Sería conveniente que en el propio RGC se estableciera el procedimiento concreto para calcular las limitaciones en aquellos casos en que existan varios métodos de cálculo posibles, como es el caso de la agudeza auditiva.

- En los CRC para cumplir con las exigencias recogidas en el Anexo IV del RGC, los médicos encargados de emitir el informe de aptitud para obtener la licencia de conducción, han de rellenar el modelo de historia clínica que aparece en el Anexo III del RD 170/2010. En este Anexo, en relación con la agudeza auditiva en las hipoacusias, se indica el procedimiento a utilizar para el cálculo del índice de pérdida combinada, de acuerdo con umbrales de audición en las frecuencias de 500, 1000, 2000 y $4000 \mathrm{~Hz}$.

- Aunque desde un punto de vista estrictamente normativo, el resultado de la valoración de la aptitud para conducir según los requisitos exigidos en el RGC no es vinculante para la actuación de los médicos inspectores que evalúen la capacidad laboral, puesto que la imposibilidad de renovación de la licencia de conducción en estos casos viene condicionada por causas médicas, puede entenderse coherente respetar esos criterios y proteger al trabajador como incapacitado.

- Si aceptamos que para la valoración de la capacidad laboral en determinadas profesiones (aquellas que incluyen entre sus requerimientos fundamentales la conducción de vehículos de motor), uno de los factores a considerar es lo dispuesto en el Anexo IV del RGC, sería importante que hubiera consenso en la interpretación del mismo buscando la homogeneización y armonización de criterios entre los distintos agentes con competencias en la valoración de incapacidades, de cara a seguir unos criterios homogéneos en todo el territorio nacional y disminuir la variabilidad interobservador.

- Son diversos los procedimientos validados para el cálculo del umbral auditivo o porcentaje de pérdida auditiva en las hipoacusias, cuya aplicación puede proporcionar resultados diferentes. Puesto que el Reglamento de los CRC, aprobado por RD 170/2010, 
establece como preceptivo un método para este cálculo, entendemos que debe ser este método el que se utilice para determinar los requisitos establecidos en el Anexo IV del RGC.

\section{REFERENCIAS BIBLIOGRÁFICAS}

1. Real Decreto Legislativo 1/1994 por el que se aprueba el Texto Refundido de la Ley General Seguridad Social.

2. Real Decreto 1971/1999, de 23 de diciembre, de procedimiento para el reconocimiento, declaración y calificación del grado de discapacidad.

3. Real Decreto $286 / 2006$, de 10 de marzo, sobre la protección de la salud y la seguridad de los trabajadores contra los riesgos relacionados con la exposición al ruido.

4. Real Decreto 1299/2006 por el que se aprueba el cuadro de enfermedades profesionales en el sistema de la Seguridad Social, y se establecen criterios para su notificación y registro.

5. Real Decreto $818 / 2009$, de 8 de mayo, por el que se aprueba el Reglamento General de Conductores, y más concretamente su anexo IV,

6. Orden PRE/2356/2010, de 3 de septiembre, por la que se establecen las aptitudes psicofísicas requeridas para obtener o prorrogar la vigencia del permiso o de la licencia de conducción.

7. Real Decreto $170 / 2010$, de 19 de febrero, por el que se aprueba el Reglamento de centros de reconocimiento destinados a verificar las aptitudes psicofísicas de los conductores.

8. Orden ESS/66/2013, de 28 de enero, por la que se actualizan las cantidades a tanto alzado de las indemnizaciones por lesiones, mutilaciones y deformidades de carácter definitivo y no invalidantes. Recomendación biap 02/1.

9. Lorente Tortosa J. M. La audición en la valoración del daño corporal. Cuadernos de valoración. Tomo II-año 2004-N. ${ }^{\circ} 3$.

10. Protocolo de vigilancia sanitaria específica de los/las trabajadores/as expuestos al ruido. Consejo Interterritorial. Ministerio de Sanidad, Servicios Sociales e Igualdad.

11. Sánchez Galán L, Rodríguez Ortiz de Salazar B. Revisión médico legal y estado actual de la evaluación médica de la hipoacusia profesional en el sistema de la Seguridad Social.

12. Medicina y seguridad en el trabajo de la Escuela Nacional de Medicina del Trabajo. Volumen LI. N. 198 (2005).

13. Sánchez Galán L, Jardón Dato E, Cubero Rivera MT. Otorrinolaringología. En: VVAA Manual de actuación para médicos inspectores del INSS (3. ${ }^{a}$ edición). Instituto Nacional de la Seguridad Social. Madrid, 2014.

14. VVAA. Guía de Valoración Profesional (3. edición). Instituto Nacional de la Seguridad Social. Madrid, 2014.



